\title{
Double Zernike expansion of the optical aberration function from its power series expansion
}

\author{
Joseph J. M. Braat ${ }^{1, *}$ and Augustus J. E. M. Janssen ${ }^{2}$ \\ ${ }^{1}$ Optics Research Group, Faculty of Applied Sciences, Technical University Delft, Lorentzweg 1, \\ 2628 CJ Delft, The Netherlands \\ ${ }^{2}$ Department of Mathematics and Computer Science, Eindhoven University of Technology, P.O. Box 513, \\ 5600 MB Eindhoven, The Netherlands \\ *Corresponding author: j.j.m.braat@tudelft.nl
}

Received April 16, 2013; accepted April 23, 2013;

posted April 26, 2013 (Doc. ID 188903); published May 28, 2013

\begin{abstract}
Various authors have presented the aberration function of an optical system as a power series expansion with respect to the ray coordinates in the exit pupil and the coordinates of the intersection point with the image field of the optical system. In practical applications, for reasons of efficiency and accuracy, an expansion with the aid of orthogonal polynomials is preferred for which, since the 1980s, orthogonal Zernike polynomials have become the reference. In the literature, some conversion schemes of power series coefficients to coefficients for the corresponding Zernike polynomial expansion have been given. In this paper we present an analytic solution for the conversion problem from a power series expansion in three or four dimensions to a double Zernike polynomial expansion. The solution pertains to a general optical system with four independent pupil and field coordinates and to a system with rotational symmetry in which case three independent coordinate combinations have to be considered. The conversion of the coefficients is analytically in closed form and the result is independent of a specific sampling scheme or sampling density as this is the case for the commonly used least squares fitting techniques. Computation schemes are given that allow the evaluation of coefficients of arbitrarily high order in pupil and field coordinates. (C) 2013 Optical Society of America

OCIS codes: $\quad$ (080.0080) Geometric optics; (080.1005) Aberration expansions; (110.0110) Imaging systems. http://dx.doi.org/10.1364/JOSAA.30.001213
\end{abstract}

\section{INTRODUCTION}

Soon after the discovery of photography, optical aberration theory was developed systematically by Seidel [1]. The optical aberration is given in terms of the transverse aberration components in the image plane, for rays that are labeled by means of their (Cartesian) coordinates in the exit pupil plane of the optical system. The optical system possesses symmetry of revolution, and the aberration is given with respect to the perfect (paraxial) imaging condition. Seidel's theory requires paraxial input data but enables the calculation of path-length differences (wavefront aberration) between rays up to the fourth-order in the coordinates of the ray intersection points with the optical surfaces and the exit pupil plane and the coordinates of the (ideal) image point pertaining to a pencil of rays. Later authors have increased the order of the approximation. We mention the work of Schwarzschild [2] for the fifth-order theory. Further developments are found in [3] and in the extensive work by Buchdahl [4] and Rimmer [5]. The calculation of aberration coefficients up to orders as high as 11 for the spherical aberration has been demonstrated. This work from 1950s to 1960 s has been reconsidered and modernized in more recent years using formal iteration schemes and computer algebra. In theory, arbitrarily high orders of approximation of the aberration function of an optical system can be reached nowadays as it has been shown in [묵].

The aberration analysis described above is based on the tracing of rays through an optical system with increased precision depending on the order to which intersection points with the optical surfaces and ray directions are calculated. In parallel, the Hamiltonian approach to optical system analysis [8] was further developed [9-11]. The aberration function is obtained in terms of wavefront (path-length) deviations between ray pairs in the object and image space. The precision with which the deviations are calculated is improved by inserting higher orders of approximation in the path-length expressions [12-14]. As in the case of Seidel-based aberration analysis, the Hamiltonian path-length deviations are written as power series expansions of the coordinates (ray direction, intersection point) in object space, in all intermediate spaces and in the image space. The elimination of the intermediate ray variables is the more complicated part of the Hamiltonian approach to optical aberration theory.

Originally, Seidel's theory was in terms of the transverse aberration components that are of third-order in the ray coordinates. A gradual transition from transverse aberration analysis to wave front aberration analysis can be observed in the past. A basic impetus to the use of wavefront aberration for optical system characterization has been given by Hopkins [15]. The ray-optics based theory of optical systems, well represented by the contents of Conrady's books [16], has been "translated" by Hopkins into the wave aberration domain. The continuous quality refinement of optical systems has pushed the analysis toward more accurate wavefront aberration analysis; as an example we mention [17] that focuses on sixth-order wavefront aberration coefficients. 
In parallel to the theoretical developments to higher order, the measurement of wavefront deviation has been substantially refined in recent years. Very high order dependencies are included in measurements to represent not only "low frequency" aberrations but also surface-induced deviations with higher spatial frequency variation. Examples of these higher order effects, both in modeling and in measurement, can be found in high-resolution projection lenses for lithography and in large astronomical telescopes. In the case of the lithographic projection lenses, extremely tight requirements on distortion and field curvature ask for a very accurate representation of the optical aberration function with respect to aperture and field coordinates. Higher order coefficients are also needed when the pupil domain, scaled to the unit circle, shows some discontinuous delimitation (vignetting of the off-axis imaging pencils, central obstruction of a telescope, hexagonal subapertures, etc.).

In general, function expansions with the aid of monomials, in most cases comprising coefficients with alternating signs of the binomial type, suffer from loss of digits. If it is possible to obtain an expansion of the same function with respect to orthogonal polynomials, this problem is drastically reduced. The numerical examples that are given in this paper will illustrate the benefit of such a change from monomial expansion to expansion with Zernike polynomials. It follows from the preceding discussion that a comprehensive coefficient conversion scheme from a power series expansion to a double Zernike expansion in pupil and field coordinates up to very high orders, typically 50-100 and even higher, would be a useful tool for the optical scientist and engineer. Efforts in this direction $[\underline{18}, \underline{19}]$ were limited to a single Zernike expansion. Moreover, the computation schemes do not support higher orders than typically 20-40. A numerical breakdown occurs in the proposed expressions for the converted coefficients due to the extensive use of factorials.

In what follows we limit ourselves to wavefront analysis of an optical system. Wavefront aberration functions in terms of power series expansions are available, but such an expansion is not optimum. A double Zernike expansion of the aberration function or of the transverse aberration components is proposed in [20]. Such an expansion has been further studied in [21] and applied to high-quality microlithographic projection lenses for the global optimization of the aberration function [22]. The optical aberration function has equally been used to study the distribution over the image field of the aberrations of circularly symmetric optical systems whose quality is affected by decentered or tilted surfaces, prismatic effects, cylindrical surface deformations, etc. Pioneering work in this field can be found in [23] with subsequent work reported in [24-27]. The power series expansion based analysis of perturbed optical systems has been translated to the Zernike framework in [28], for the lower image field dependencies up to an order of 6 .

An expansion with respect to Zernike polynomials on the exit pupil plane and image plane coordinates is more appropriate and yields better results regarding efficiency and accuracy than the corresponding power series expansion. Especially in the case of a wavefront reconstruction or retrieval operation, efficiency is greatly improved when orthogonal polynomials are used for the representation of the aberration function. In Section 2 we briefly present the wavefront aberration function for a general optical system and for the frequently occurring system with symmetry of revolution. In Section 3 we present the conversion scheme for an optical system with symmetry of revolution. We obtain, in an orderly and systematic manner, the double Zernike expansion coefficients of the pupil-field aberration function from the coefficients of the power series expansion in a closed form. The expressions allow the calculation of arbitrarily high orders and satisfy the needs of present-day and future scientists and engineers who work on high-quality imaging optics. Calculation of high-order Zernike polynomials $Z_{n}^{m}(\rho, \theta)$ is unreliable when resorting to the standard power series expressions for the radial polynomials. When applying a recursive scheme, this computational problem is effectively removed $[29,30]$. Section $\underline{4}$ focuses on the more general case of a system without any symmetry. Some examples of coefficient conversion are given in Section 5 . The paper ends with some conclusions on the type of functions that can be handled and on the practical implementation of the method.

\section{OPTICAL ABERRATION FUNCTION}

In Fig. 1 we sketch an oblique pencil of rays that leaves an optical system toward a paraxial image point $A_{1}$. For a particular ray through a point $P_{1}(X, Y)$ in the exit pupil plane the transverse aberration components $\left(\delta x_{A}, \delta y_{A}\right)$ are calculated. Simultaneously, by optical path-length calculations along a ray or from a Hamiltonian characteristic function, we obtain the path-length difference of a particular ray with respect to the reference ray. The reference ray generally is the ray that intersects the center of the diaphragm of the optical system; it passes not necessarily through $E_{1}$, the center of the exit pupil, because of the presence of aberrations in the imaging of the pupils. We define an optical aberration function by the expression

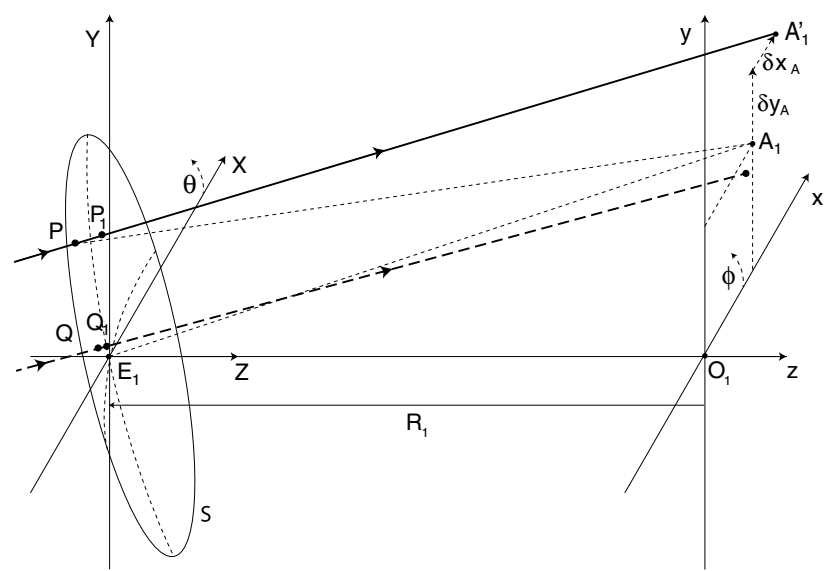

Fig. 1. Ray propagation from the exit pupil plane to the image plane An aberrated aperture ray intersects the reference sphere through $E_{1}$ in the exit pupil in the point $P$ with coordinates $\left(X_{P}, Y_{P}, Z_{P}\left(X_{P}, Y_{P}\right)\right)$. The reference ray (dashed in the figure) intersects the reference sphere in $Q$. The position of the perfect image point is $A_{1}$. The aberrated ray intersects the pupil plane in the point $P_{1}(X, Y)$ and the image plane in the point $A_{1}^{\prime}$, with coordinates $\left(x_{A}+\delta x_{A}, y_{A}+\delta y_{A}\right)$. The distance from the center $O_{1}$ of the image plane to the center $E_{1}$ of the exit pupil is $R_{1}$, negative in the figure. $P A_{1}=E_{1} A_{1}$ is the radius of the reference sphere $S$, centered on $A_{1}$, that is associated with the particular oblique imaging pencil issued from an object point $A_{0}$ (not shown in the figure). 


$$
W(X, Y, x, y)=\left[A_{0} P\right]-\left[A_{0} Q\right] .
$$

$A_{0}$ is the object point for the pencil of rays (not shown in the figure), and $P$ and $Q$ are the intersection points of the general aperture ray and the reference ray with the reference sphere $S$ associated with the (perfect) image point $A_{1}$. The aberration function $W$ is defined with respect to the coordinates $(X, Y)$ of the intersection point $P_{1}$ of the general aperture ray with the exit pupil plane through $E_{1}$ and the image plane coordinates $(x, y)$ of the hypothetical perfect image point $A_{1}$ produced by the object point $A_{0}$. The aberration function of a general optical system is written in terms of the Cartesian pupil and field coordinates [15],

$$
\begin{aligned}
W(X, Y ; x, y) & =\sum a_{n m l k}^{\prime} X^{n} Y^{m} x^{l} y^{k} \\
& =\sum a_{n m l k}^{\prime} \rho_{P_{1}}^{n+m} r_{f}^{l+k} \cos ^{n} \theta \sin ^{m} \theta \cos ^{l} \phi \sin ^{k} \phi
\end{aligned}
$$

with $\rho_{P_{1}}$ and $r_{f}$ the radial coordinates with respect to the origins $E_{1}$ and $O_{1}$, respectively, of pupil and field coordinates; see Fig. 1 . The azimuthal angle $\theta$ is measured with respect to the $X$ axis of the right-handed $(X, Y, Z)$-coordinate system in the pupil plane; this angle is positive when the azimuthal orientation is rotated clockwise when viewing in the direction of the positive $Z$ axis. A comparable argument holds for the azimuth $\phi$ in the image plane.

For an optical system with an axis of rotational symmetry, the aberration function depends on coordinate combinations that are invariant with respect to a rotation around the axis $O_{0} O_{1}$ of the optical system $\left(O_{0}\right.$ is the center of the object plane). Defining the pupil and field vectors $\rho_{P_{1}}=(X, Y)=\rho_{P_{1}}$ $(\cos \theta, \sin \theta)$ and $\boldsymbol{r}_{f}=(x, y)=r_{f}(\cos \phi, \sin \phi)$, the rotation invariant combinations are $\boldsymbol{\rho}_{P_{1}} \cdot \boldsymbol{\rho}_{P_{1}}, \boldsymbol{r}_{f} \cdot \boldsymbol{r}_{f}$, and $\boldsymbol{\rho}_{P_{1}} \cdot \boldsymbol{r}_{f}$. The power series expansion of the aberration function is then given by

$$
\begin{aligned}
& W\left\{\rho_{P_{1}}^{2}, r_{f}^{2}, \rho_{P_{1}} r_{f} \cos (\theta-\phi)\right\} \\
& \quad=\sum a_{n m l}^{\prime}\left(\rho_{P_{1}}^{2}\right)^{n}\left(r_{f}^{2}\right)^{l}\left[r_{f} \rho_{P_{1}} \cos (\theta-\phi)\right]^{m} .
\end{aligned}
$$

The number of terms in each wavefront expansion needs a short discussion. In optical aberration theory, the terms that purely depend on the image plane coordinates are generally omitted [31]. They influence the phase of the optical disturbance in the image plane but are of no relevance for the calculation of the image intensity. With this restriction, the total number of terms of a certain order $N$ is given by

$$
N_{n s}=\frac{N(N+1)(N+5)}{6}, \quad N_{r s}=\frac{N(N+6)}{8}
$$

with $N_{n s}$ applying to the general system and $N_{r s}$ to a system with rotational symmetry. In the latter case, $N$ is restricted to even and nonnegative integer values. The power series expansions of Eqs. (2) and (3) will be converted into Zernike expansions in the next sections. We first address the aberration function of Eq. (3) because it applies to the common optical systems that possess rotational symmetry in their as-designed geometry. In the next step we address the problem of the general optical system without any symmetry, either because a system with rotational symmetry suffers from manufacturing errors or because the design itself lacks any symmetry property (e.g., "free-form" optics).

\section{ZERNIKE EXPANSION FOR AN OPTICAL SYSTEM WITH ROTATIONAL SYMMETRY}

To find the Zernike coefficients of the power expansion according to Eq. (3), we calculate the inner product of a specific term with coefficient $a_{n m l}^{\prime}$ with a (complex) double Zernike polynomial in pupil and field coordinates. The complex Zernike polynomials $Z_{n}^{m}(\rho, \theta)=R_{n}^{|m|}(\rho) \exp (\operatorname{im} \theta)$ are chosen because they allow a much simpler administration than those with separate cosine and sine polynomials. We have for any pupil function $f(\rho, \theta)$ the orthogonal expansion

$$
f(\rho, \theta)=\sum_{n, m} c_{n m} R_{n}^{|m|}(\rho) \exp (i m \theta)
$$

with $c_{n m}$ generally complex. The normalization of the Zernike polynomials is such that

$$
\int_{0}^{2 \pi} \int_{0}^{1}\left|Z_{n}^{m}(\rho, \theta)\right|^{2} \rho \mathrm{d} \rho \mathrm{d} \theta=\frac{\pi}{n+1} .
$$

Thus the $c_{n m}$ in Eq. (5) are given by

$$
c_{n m}=\frac{n+1}{\pi} \int_{0}^{2 \pi} \int_{0}^{1} f(\rho, \theta) Z_{n}^{m *}(\rho, \theta) \rho \mathrm{d} \rho d \theta .
$$

The cosine and sine coefficients of the Zernike expansion of a general complex function follow from

$$
a_{c}=\left(c_{n m}+c_{n,-m}\right), \quad a_{s}=+i\left(c_{n m}-c_{n,-m}\right) .
$$

In the case of a real function we have the special property $c_{n,-m}=c_{n m}^{*}$.

Before calculating the inner product we have to normalize the radial coordinates in pupil and field to unity and obtain an expansion as in Eq. (3) with new unprimed expansion coefficients $a_{n m l}$. We have the following double Zernike expansion of the aberration function:

$$
\begin{aligned}
& W(\rho, r ; \theta, \phi) \\
& \quad=\sum_{n_{1}, n_{2} ; m_{1}, m_{2}} c_{n_{1} n_{2} m_{1} m_{2}} R_{n_{1}}^{\left|m_{1}\right|}(\rho) R_{n_{2}}^{\left|m_{2}\right|}(r) \exp \left[i\left(m_{1} \theta+m_{2} \phi\right)\right]
\end{aligned}
$$

with $\rho$ and $r$ the normalized versions of the real-space radial coordinates $\rho_{P}$ and $r_{f}$.

To find the complete set of Zernike coefficients, we proceed in two steps. We first select a general term with index $\mathrm{nlm}$ from the power series expansion and calculate the inner product with a general Zernike term from the expansion of Eq. (9). The inner product is denoted by $I_{n l m}^{n_{1} n_{2} m_{1} m_{2}}$ with $n_{1} n_{2} m_{1} m_{2}$ the indices of the Zernike polynomial; the inner product yields nonzero values for certain index combinations $n l m m$ and $n_{1} n_{2} m_{1} m_{2}$, depending also on the properties of the aberration function, for instance, the presence of circular symmetry. The complete Zernike expansion then follows from a summation of the coefficients over all possible terms $a_{n l m}$ of the power series. 
For the inner product of a single term from the power series with $Z_{n_{1}}^{m_{1}}(\rho, \theta) Z_{n_{2}}^{m_{2}}(r, \phi)$ we write

$$
\begin{aligned}
I_{n l m}^{n_{1} n_{2} m_{1} m_{2}}= & \int_{0}^{1} \int_{0}^{1} \int_{0}^{2 \pi} \int_{0}^{2 \pi} \rho^{2 n+m} r^{2 l+m} \cos ^{m}(\theta-\phi) R_{n_{1}}^{\left|m_{1}\right|}(\rho) R_{n_{2}}^{\left|m_{2}\right|}(r) \\
& \times \exp \left[-i\left(m_{1} \theta+m_{2} \phi\right)\right] \rho r \mathrm{~d} \rho \mathrm{d} r \mathrm{~d} \theta \mathrm{d} \phi
\end{aligned}
$$

Writing $\cos (\theta-\phi)$ as $(\exp [i(\theta-\phi)]+\exp [-i(\theta-\phi)]) / 2$ and using Newton's binomial formula, we expand

$$
\cos ^{m}(\theta-\phi)=\frac{1}{2^{m}} \sum_{j=0}^{m}\left(\begin{array}{c}
m \\
j
\end{array}\right) \exp [i(m-2 j)(\theta-\phi)]
$$

Therefore

$$
\begin{aligned}
& \int_{0}^{2 \pi} \int_{0}^{2 \pi} \cos ^{m}(\theta-\phi) \exp \left[-i\left(m_{1} \theta+m_{2} \phi\right)\right] \mathrm{d} \theta \mathrm{d} \phi \\
& =\frac{1}{2^{m}} \sum_{j=0}^{m}\left(\begin{array}{c}
m \\
j
\end{array}\right) \int_{0}^{2 \pi} \int_{0}^{2 \pi} \exp \left[+i\left(m-m_{1}-2 j\right) \theta\right] \\
& \left.\quad \times \exp \left[-i\left(m+m_{2}-2 j\right) \phi\right)\right] \mathrm{d} \theta \mathrm{d} \phi \\
& =\frac{4 \pi^{2}}{2^{m}} \sum_{j=0}^{m}\left(\begin{array}{c}
m \\
j
\end{array}\right) \delta_{m-m_{1}-2 j} \delta_{m+m_{2}-2 j}
\end{aligned}
$$

with $\delta_{n}=1$ for $n=0$ and 0 otherwise. Thus, the right-hand side of Eq. (12) equals

$$
\begin{cases}\frac{4 \pi^{2}}{2^{m}}\left(\begin{array}{c}
m \\
\frac{m-\left|m_{1}\right|}{2}
\end{array}\right) & m_{1}=-m_{2} ; m-\left|m_{1,2}\right| \text { even and nonnegative } \\
0 & \text { otherwise. }\end{cases}
$$

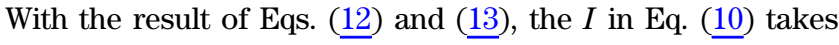
the form

$$
\begin{aligned}
& I_{n l m}^{n_{1} n_{2} m_{1} m_{2}} \\
& \quad=\frac{4 \pi^{2}}{2^{m}}\left(\begin{array}{c}
m \\
\frac{m-\left|m_{1}\right|}{2}
\end{array}\right) \int_{0}^{1} \rho^{2 n+m} R_{n_{1}}^{\left|m_{1}\right|}(\rho) \rho \mathrm{d} \rho \int_{0}^{1} r^{2 l+m} R_{n_{2}}^{\left|m_{1}\right|}(r) r \mathrm{~d} r .
\end{aligned}
$$

Equation (14) illustrates that, for a rotationally symmetric optical system, the Zernike expansion possesses three independent indices $n_{1}, n_{2}$, and $m_{1}=m_{2}$ because of a coupling between the azimuthal indices of the pupil and field polynomials.

The integral over $\rho$ has been discussed in [32] (see also Appendix $\underline{\text { A}}$ ), and we obtain with $p_{1}=\left(n_{1}-\left|m_{1}\right|\right) / 2$

$$
\begin{aligned}
J_{n m}^{n_{1} m_{1}} & =\int_{0}^{1} \rho^{2 n+m} R_{n_{1}}^{\left|m_{1}\right|}(\rho) \rho \mathrm{d} \rho \\
& =\frac{\frac{1}{2}\left(n+\frac{m-\left|m_{1}\right|}{2}\right) !\left(n+\frac{m+\left|m_{1}\right|}{2}\right) !}{\left(n+\frac{m-\left|m_{1}\right|}{2}-p_{1}\right) !\left(n+\frac{m+\left|m_{1}\right|}{2}+p_{1}+1\right) !}
\end{aligned}
$$

which is nonvanishing only when $n_{1}=\left|m_{1}\right|,\left|m_{1}\right|+2, \ldots$, $2 n+m$. For the integral over $r$ there is a similar result, viz. $J_{l m}^{n_{2} m_{1}}$ with $p_{2}=\left(n_{2}-\left|m_{1}\right|\right) / 2$ instead of $p_{1}$.
We then obtain for a single power series term by Eq. (7) the following Zernike polynomial expansion:

$$
\begin{aligned}
& \rho^{2 n+m} r^{2 l+m} \cos ^{m}(\theta-\phi) \\
& \quad=\sum_{n_{1}=0}^{2 n+m} \sum_{n_{2}=0}^{2 l+m} \sum_{m_{1}=-m}^{m} b_{n m l}^{n_{1} n_{2} m_{1}} R_{n_{1}}^{\left|m_{1}\right|}(\rho) R_{n_{2}}^{\left|m_{1}\right|}(r) \exp \left[i m_{1}(\theta-\phi)\right]
\end{aligned}
$$

with

$$
\begin{aligned}
b_{n m l}^{n_{1} n_{2} m_{1}}= & \frac{4\left(n_{1}+1\right)\left(n_{2}+1\right)}{2^{m}}\left(\begin{array}{c}
m \\
\frac{m-\left|m_{1}\right|}{2}
\end{array}\right) J_{n m}^{n_{1} m_{1}} J_{l m}^{n_{2} m_{1}} \\
= & \frac{\left(n_{1}+1\right)\left(n_{2}+1\right)}{2^{m}\left(\begin{array}{c}
m \\
\frac{m-\left|m_{1}\right|}{2}
\end{array}\right)} \\
& \times \frac{\left(n+\frac{m-\left|m_{1}\right|}{2}\right) !\left(n+\frac{m+\left|m_{1}\right|}{2}\right) !}{\left(n+\frac{m-\left|m_{1}\right|}{2}-p_{1}\right) !\left(n+\frac{m+\left|m_{1}\right|}{2}+p_{1}+1\right) !} \\
& \times \frac{\left(l+\frac{m-\left|m_{1}\right|}{2}\right) !\left(l+\frac{m+\left|m_{1}\right|}{2}\right) !}{\left(l+\frac{m-\left|m_{1}\right|}{2}-p_{2}\right) !\left(l+\frac{m+\left|m_{1}\right|}{2}+p_{2}+1\right) !}
\end{aligned}
$$

The numbers $p_{1}$ and $p_{2}$ are $\left(n_{1}-\left|m_{1}\right|\right) / 2$ and $\left(n_{2}-\left|m_{1}\right|\right) / 2$, respectively. The summation range for $m_{1}$ is restricted to the following values: $m-\left|m_{1}\right|$ is even and nonnegative and $\left|m_{1}\right| \leq \min \left(n_{1}, n_{2}\right)$.

For the complete power series expansion with index ranges $0 \leq n \leq N_{p}, 0 \leq l \leq L_{p}$, and $0 \leq m \leq M_{p}$, we obtain the Zernike expansion

$$
\begin{aligned}
& \sum_{n l m} a_{n l m} \rho^{2 n+m} r^{2 l+m} \cos ^{m}(\theta-\phi) \\
& =\sum_{n_{1}=0}^{2 N_{p}+M_{p}} \sum_{n_{2}=0}^{2 L_{p}+M_{p}} \sum_{m_{1}=-M_{p}}^{M_{p}}\left\{\sum_{n l m} a_{n l m} b_{n m l}^{n_{1} n_{2} m_{1}}\right\} R_{n_{1}}^{\left|m_{1}\right|}(\rho) R_{n_{2}}^{\left|m_{1}\right|}(r) \\
& \quad \times \exp \left[i m_{1}(\theta-\phi)\right]=\sum_{n_{1} n_{2} m_{1}} c_{n_{1} n_{2} m_{1}} R_{n_{1}}^{\left|m_{1}\right|}(\rho) R_{n_{2}}^{\left|m_{1}\right|}(r) \\
& \quad \times \exp \left[i m_{1}(\theta-\phi)\right]
\end{aligned}
$$

with $c_{n_{1} n_{2} m_{1}}$ the new coefficients of the Zernike polynomial expansion and the series over $m_{1}$ restricted as in Eq. (16).

The expression for the $b$ coefficients in (16) is in a closed form with a well-defined, limited number of terms. As is pointed out in Appendix A, the two expressions for the radial integrals can be written in a form that circumvents the use of factorials and leads to a reliable product expression with multiplication factors $\leq 1$. The expression in Eq. (13) for the azimuthal integral can be evaluated without problems when $m$ is not too large (say, $m \leq 20$ ). Alternatively, a reliable, on DFT's based, method is given in Appendix $\underline{B}$.

\section{ZERNIKE EXPANSION FOR A GENERAL OPTICAL SYSTEM}

To find the Zernike coefficients of the power expansion according to Eq. (2), we proceed along the same lines as in the previous section. We normalize the radial coordinates in pupil and field to unity and obtain the unprimed expansion coefficients $a_{n m l k}$. The inner product of a general term of the power series expansion with coefficient $a_{n m l k}$ and a double 
Zernike polynomial with indices $n_{1} m_{1} n_{2} m_{2}$ is given by

$$
\begin{aligned}
I_{n l m}^{n_{1} n_{2} m_{1} m_{2}}= & \int_{0}^{1} \int_{0}^{1} \int_{0}^{2 \pi} \int_{0}^{2 \pi} \rho^{n+m} r^{l+k} \\
& \times \cos ^{n} \theta \sin ^{m} \theta \cos ^{l} \phi \sin ^{k} \phi R_{n_{1}}^{\left|m_{1}\right|}(\rho) R_{n_{2}}^{\left|m_{2}\right|}(r) \\
& \times \exp \left[-i\left(m_{1} \theta+m_{2} \phi\right)\right] \rho r \mathrm{~d} \rho \mathrm{d} r \mathrm{~d} \theta \mathrm{d} \phi .
\end{aligned}
$$

The part of the inner product that is related to an azimuthal integration over $\theta$ in Eq. (18) is given by

$$
\begin{aligned}
I_{n m}^{m_{1}}= & \int_{0}^{2 \pi} \cos ^{n} \theta \sin ^{m} \theta \exp \left(-i m_{1} \theta\right) \mathrm{d} \theta \\
= & \frac{1}{2^{n+m} i^{m}} \sum_{j_{1}=0}^{n} \sum_{j_{2}=0}^{m}\left(\begin{array}{c}
n \\
j_{1}
\end{array}\right)\left(\begin{array}{c}
m \\
j_{2}
\end{array}\right)(-1)^{j_{2}} \\
& \times \int_{0}^{2 \pi} \exp \left[i\left(n-2 j_{1}\right) \theta+i\left(m-2 j_{2}\right) \theta\right] \exp \left(-i m_{1} \theta\right) \mathrm{d} \theta \\
= & \frac{2 \pi}{2^{n+m} i^{m}} \sum_{j_{1}=0}^{n} \sum_{j_{2}=0}^{m}\left(\begin{array}{c}
n \\
j_{1}
\end{array}\right)\left(\begin{array}{c}
m \\
j_{2}
\end{array}\right)(-1)^{j_{2}} \delta_{n+m-m_{1}-2 j_{1}-2 j_{2}} .
\end{aligned}
$$

This is nonvanishing only when $n+m-m_{1}$ is an even integer between 0 and $2(n+m)$, i.e., when $n+m-\left|m_{1}\right|$ is even and nonnegative. In that case we compute

$$
I_{n m}^{m_{1}}=\frac{2 \pi}{2^{n+m} i^{m}} \sum_{j}(-1)^{j}\left(\begin{array}{c}
m \\
j
\end{array}\right)\left(\begin{array}{c}
n \\
\frac{n+m-m_{1}}{2}-j
\end{array}\right)
$$

with a nonempty summation range over all integer $j \geq$ $\max \left[0,\left(m-n-m_{1}\right) / 2\right]$ and $\leq \min \left[m,\left(n+m-m_{1}\right) / 2\right]$. The integral over $\phi$ in Eq. (18) is given in a similar fashion as $I_{l k}^{m_{2}}$. The expression in Eq. (20) can be evaluated without problems when $n$ and $m$ are not too large. Otherwise, a reliable method is given in Appendix $\underline{B}$.

For the integration over the radial coordinate $\rho$ in Eq. (18) we again use the result of Appendix $\underline{A}$,

$$
\begin{aligned}
K_{n m}^{n_{1} m_{1}} & =\int_{0}^{1} \rho^{n+m} R_{n_{1}}^{\left|m_{1}\right|}(\rho) \rho \mathrm{d} \rho \\
& =\frac{\frac{1}{2}\left(\frac{n+m-\left|m_{1}\right|}{2}\right) !\left(\frac{n+m+\left|m_{1}\right|}{2}\right) !}{\left(\frac{n+m-\left|m_{1}\right|}{2}-p_{1}\right) !\left(\frac{n+m+\left|m_{1}\right|}{2}+p_{1}+1\right) !}
\end{aligned}
$$

with $p_{1}=\left(n_{1}-\left|m_{1}\right|\right) / 2$. We have for $K$ in Eq. (21) a nonzero result only if $n_{1}=\left|m_{1}\right|,\left|m_{1}\right|+2, \ldots,(n+m)$. Similarly, the integral over $r$ in Eq. (18) yields $K_{l k}^{n_{2} m_{2}}$ with $p_{2}=\left(n_{2}-\left|m_{2}\right|\right) / 2$ instead of $p_{1}$, and this is nonzero only if $n_{2}=\left|m_{2}\right|$, $\left|m_{2}\right|+2, \ldots,(l+k)$.

Integration over the four variables of the general expansion according to Eq. (4) yields for a single term in the power series expansion

$$
\begin{aligned}
& \rho^{n+m} r^{l+k} \cos ^{n} \theta \sin ^{m} \theta \cos ^{l} \phi \sin ^{k} \phi \\
& =\sum_{n_{1}=0}^{M} \sum_{n_{2}=0}^{K} \sum_{m_{1}=-M}^{M} \sum_{m_{2}=-K}^{K} b_{n m l k}^{n_{1} n_{2} m_{1} m_{2}} R_{n_{1}}^{\left|m_{1}\right|}(\rho) R_{n_{2}}^{\left|m_{2}\right|}(r) \\
& \quad \times \exp \left[i\left(m_{1} \theta+m_{2} \phi\right)\right]
\end{aligned}
$$

with $M=n+m$ and $K=l+k$. The coefficients $b_{n m l k}^{n_{1} n_{2} m_{1} m_{2}}$ and the inner product $I_{n m l k}^{n_{1} n_{2} m_{1} m_{2}}$ are related through the expression (7),

$$
\begin{aligned}
b_{n m l k}^{n_{1} n_{2} m_{1} m_{2}} & =\frac{\left(n_{1}+1\right)\left(n_{2}+1\right)}{\pi^{2}} I_{n m l k}^{n_{1} n_{2} m_{1} m_{2}} \\
& =\frac{\left(n_{1}+1\right)\left(n_{2}+1\right)}{\pi^{2}} I_{n m}^{m_{1}} I_{l k}^{m_{2}} K_{n m}^{n_{1} m_{1}} K_{l k}^{n_{2} m_{2}} .
\end{aligned}
$$

The complete Zernike expansion is then given by

$$
\begin{aligned}
\sum_{n m l k} & a_{n m l k} \rho^{n+m} r^{l+k} \cos ^{n} \theta \sin ^{m} \theta \cos ^{l} \phi \sin ^{k} \phi \\
= & \sum_{n_{1} n_{2} m_{1} m_{2}}\left\{\sum_{n m l k} a_{n m l k} b_{n m l k}^{n_{1} n_{2} m_{1} m_{2}}\right\} R_{n_{1}}^{\left|m_{1}\right|}(\rho) R_{n_{2}}^{\left|m_{2}\right|}(r) \\
& \times \exp \left[i\left(m_{1} \theta+m_{2} \phi\right)\right] \\
= & \sum_{n_{1} n_{2} m_{1} m_{2}} c_{n_{1} n_{2} m_{1} m_{2}} R_{n_{1}}^{\left|m_{1}\right|}(\rho) R_{n_{2}}^{\left|m_{2}\right|}(r) \times \exp \left[i\left(m_{1} \theta+m_{2} \phi\right)\right],
\end{aligned}
$$

with the coefficients $c_{n_{1} n_{2} m_{1} m_{2}}$ being the Zernike coefficients for the original power series expansion with coefficients $a_{n m l k}$.

The results that have been obtained for the coefficients of a double Zernike expansion can be directly applied to the conversion of a two-dimensional power series into a standard single Zernike expansion. With the computation scheme given above, unwieldy results can be avoided that are inherent to earlier schemes given in the literature and that produce a substantial loss of accuracy or a computational breakdown once the orders $n$ and $m$ take on values of the order of 30 or higher.

\begin{tabular}{|c|c|c|c|c|}
\hline$n_{1}$ & $n_{2}$ & $m_{1}$ & $m_{2}$ & $c_{n_{11}}$ \\
\hline 0 & 0 & 0 & 0 & $0.1428571428571429=1 / 7$ \\
\hline 2 & 0 & 0 & 0 & $0.3214285714285714=9 / 28$ \\
\hline 4 & 0 & 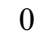 & 0 & $0.2976190476190476=25 / 84$ \\
\hline 6 & 0 & 0 & 0 & $67=1 / 6$ \\
\hline & 0 & 0 & 0 & $0.0584415584415584=9 / 154$ \\
\hline & 0 & . & 0 & $0.0119047619047619=1 / 84$ \\
\hline & 0 & 0 & 0 & $0.0010822510822511=1 / 924$ \\
\hline
\end{tabular}

\section{NUMERICAL EXAMPLES}

In this section we focus on the accuracy with which the conversion from a power series expansion to a Zernike expansion can be carried out. We also show that a Zernike polynomial expansion is much more economic regarding its number of coefficients in reproducing the original function with a certain degree of approximation. This property is demonstrated here for a single Zernike expansion but applies equally well to double Zernike expansions.

Example 1: In a first example we treat a two-dimensional example, limited to the pupil coordinates $(X, Y)$, to test the accuracy of the conversion scheme. The Zernike expansion of the monomial $\rho^{12}$ is chosen because it allows an easy analytic check of the result. The appropriate coefficients of $\left(X^{2}+Y^{2}\right)^{6}$ are inserted in the power series expansion in $(X, Y)$. With the coefficients $a_{2 n-2 j, 2 j, 0,0}=\left(\begin{array}{c}n \\ j\end{array}\right)$ for $n=6$ and $j=0,1, \ldots, n$, we obtain from Eq. (24) the Zernike coefficients $c_{2 j, 0,0,0}$ for $j=0,1, \ldots, n$,

The $c$ coefficients of the Zernike expansion reproduce the unit coefficient of $\rho^{12}$ up to the machine precision. For comparison, we have put between parentheses the quotients of integer numbers that exactly reproduce the function $\rho^{12}$. Such a test 
could equally well be carried out for an arbitrary high order of $\rho$ as the calculations do not rely on the explicit calculation of expressions including factorials of large integer numbers.

Example 2: A quadruple power series expansion is chosen according to

$$
\begin{aligned}
W(\rho, \theta, r, \phi)= & \rho^{3} r^{2}\left(\frac{1}{2} \cos ^{3} \theta+\sin ^{3} \theta\right) \cos ^{2} \phi \\
& +\rho^{4} r^{3} \cos ^{3} \theta \sin \theta \cos ^{2} \phi \sin \phi
\end{aligned}
$$

where $W$ is an example of a (real) wavefront expansion of seventh-order in pupil and field coordinates. In the converted double Zernike expansion 60 nonzero coefficients are found. The conversion process is accurate down to the machine precision and the residual error between $W$ and its Zernike expansion with maximum order equal to 7 is of this order of magnitude (15-16 significant digits when using double precision arithmetic).

Example 3: We choose

$$
f(X, Y)=\exp \{2 \pi i(u X+v Y)\}=\exp \{2 \pi i \rho w \cos (\theta-\psi)\},
$$

where $u$ and $v$ are real and $u+i v=w \exp (i \psi)$ with $w>0$ and $\psi$ real. As a pupil function, the $f$ of Eq. (27) represents an overall shift of the image, with the components of the shift vector $(u, v)$ expressed in the diffraction unit in the image plane. This example is, furthermore, relevant in the present context with respect to field-dependent distortion. When $u$ and $v$ vary as a function of the field coordinates $r$ and $\phi$, various orders of symmetrical and asymmetrical distortion are represented by the complex pupil function of Eq. (27).

The Cartesian power series representation of $f$ is given by

$$
f_{p}(X, Y)=\sum_{n, m=0}^{\infty} a_{n m} X^{n} Y^{m}, \quad \text { with } a_{n m}=\frac{(2 \pi i u)^{n}}{n !} \frac{(2 \pi i v)^{m}}{m !}
$$

The Zernike expansion of $f$ is given analytically as

$$
f_{Z}(\rho, \theta)=\sum_{n, m} c_{n m} Z_{n}^{m}(\rho, \theta)
$$

where

$$
c_{n m}=2(n+1) i^{n} \frac{J_{n+1}(2 \pi w)}{2 \pi w} \exp (-i m \psi)
$$

for integers $n, m$ such that $n-|m|$ is even and nonnegative and $u+i v=w \exp (i \psi)$ as above, and $J_{n+1}$ is the Bessel function of the first kind and of order $n+1$. This follows on computing the inner products of $f$ with Zernike circle polynomials $Z_{n}^{m}(\rho, \theta)=R_{n}^{|m|}(\rho) \exp (i m \theta)$, using

$$
\begin{aligned}
& \int_{0}^{2 \pi} \exp [2 \pi i \rho w \cos (\theta-\psi)] \exp (-i m \theta) \mathrm{d} \theta \\
& \quad=2 \pi i^{m} J_{m}(2 \pi \rho w) \exp (-i m \psi),
\end{aligned}
$$

and the basic result from the Zernike-Nijboer diffraction theory (see [29,33]),

$$
\int_{0}^{1} R_{n}^{|m|}(\rho) J_{m}(b \rho) \rho \mathrm{d} \rho=(-1)^{\frac{n-m}{2}} \frac{J_{n+1}(b)}{b} .
$$

Given $N=0,1, \ldots$, we let

$$
\begin{gathered}
f_{p}^{N}(X, Y)=\sum_{n+m \leq N} a_{n m} X^{n} Y^{m}, \\
f_{Z}^{N}=\sum_{|m| \leq n \leq N} c_{n m} Z_{n}^{m},
\end{gathered}
$$

and we let

$$
f_{p Z}^{N}=\sum_{n_{1}, m_{1}} c_{n_{1} 0 m_{1} 0}^{N} Z_{n_{1}}^{m_{1}}
$$

be the Zernike series representation of $f_{p}^{N}$ with coefficients $c_{n_{1} 0 m_{1} 0}^{N}$ obtained by the method of Section $\underline{4}$.

In Fig. 2(a) we plot the real part of the function $f$ for $\rho=1$ and $0 \leq \theta \overline{<2} \pi$ with $(u, v)=(2.5,1.2)$. In Fig. $2(\mathrm{~b})$, for $N=40$, we display on a logarithmic scale the absolute value of the difference functions

1. $\Re\left\{f(1, \theta)-f_{p}^{40}(1, \theta)\right\}$,

2. $\Re\left\{f_{p}^{40}(1, \theta)-f_{p Z}^{40}(1, \theta)\right\}$,

3. $\Re\left\{f(1, \theta)-f_{Z}^{40}(1, \theta)\right\}$,

as a function of $\theta, 0 \leq \theta<2 \pi$, i.e., $(X, Y)$ on the rim of the pupil. It is seen that both $f_{p}^{40}$ and $f_{p Z}^{40}$ provide a very poor approximation of $f$, that $f_{p}^{40}$ and $f_{p Z}^{40}$ agree up to what can be achieved $\left(10^{-9}\right)$ given the large values of $a_{n m}$, up to $10^{8}$, and the machine precision of typically $10^{-16}$. One also observes that $f_{Z}^{40}$ gives an approximation of $f$ with an error of $10^{-11}$ that is of the order of the first neglected $c_{n m}$ 's in the Zernike expansion of $f$ in Eqs. (29) and (30).

The next exercise is to show that-in this case of an analytically given $f$-using a substantially lower number of Zernike terms in Eq. (35) still can provide an approximation of $f$ of the same quality as the truncated power series $f_{p}^{N}$. Thus we let for $N_{1}=0,1, \ldots, N$

$$
f_{p Z}^{N, N_{1}}=\sum_{\left|m_{1}\right| \leq n_{1} \leq N_{1}} c_{n_{1} 0 m_{1} 0}^{N} Z_{n_{1}}^{m_{1}}
$$

i.e., we use the $c$ 's computed with $N$, but we include only those Zernike terms corresponding to degrees $\leq N_{1}$. We let $u=2.5$ and $v=1.2$ as before, and we compute for a given $N$ rms values $\delta$ of the (complex) quantities $f-f_{\text {app }}$ according to

$$
\delta=\left(\frac{1}{J} \sum_{j=1}^{J}\left|f\left\{(X, Y)_{j}\right\}-f_{\text {app }}\left\{(X, Y)_{j}\right\}\right|^{2}\right)^{1 / 2}
$$

where the $(X, Y)_{j}$ with $\left(X^{2}+Y^{2}\right)^{1 / 2} \leq 1$ are the points comprised in a square window with side lengths 2 and lying on the intersection points of a square grid (side length 0.2 ) with the central intersection point located at the arbitrarily chosen position $(X, Y)=(0.03142,-0.0783) ; J$ amounts to 79 for this sampling grid. We denote by $\delta_{1}, \delta_{2}$, and $\delta_{3}$ the $\delta$ obtained in Eq. (37) where we choose $f_{\text {app }}=f_{p}^{N}, f_{Z}^{N}$, and $f_{p Z}^{N, N_{1}}$, respectively. In Fig. 3, there is displayed the ${ }^{10} \log \delta_{1}$ and ${ }^{10} \log \delta_{2}$ (solid curve and dot-dashed curve, respectively) as a function 


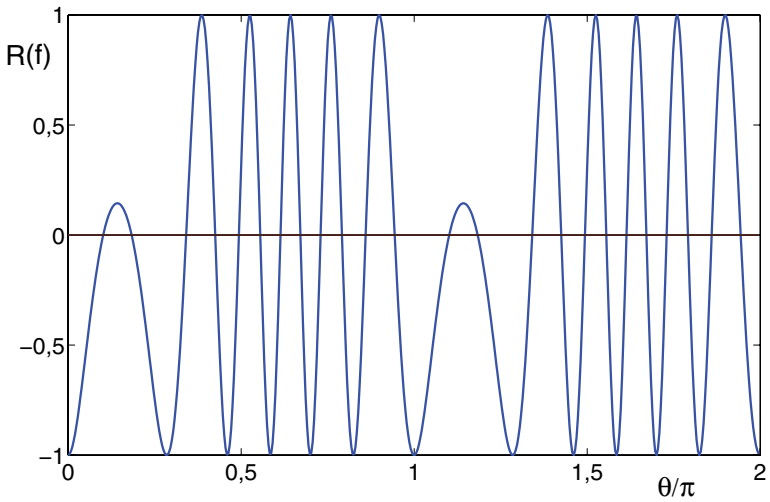

(a)

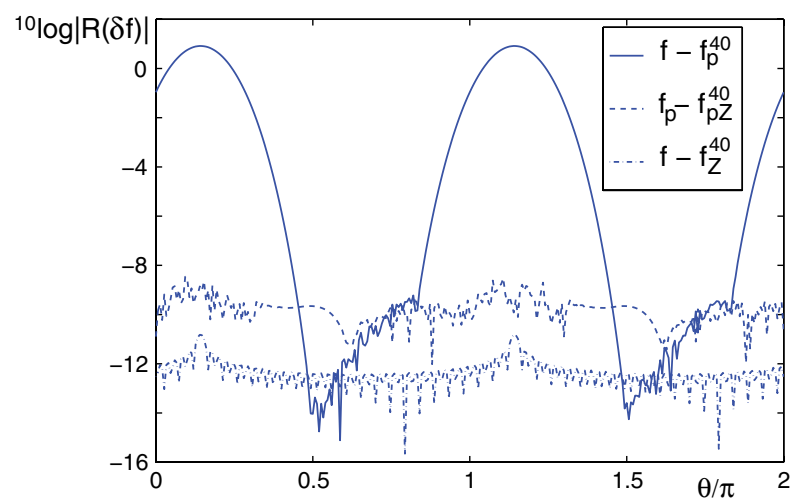

(b)

Fig. 2. Real part of the exponential function $f(\rho, \theta)$. The parameter values are $u=2.5$ and $v=1.2$. (a) $\Re\{f(X, Y)\}$ at the rim of the unit circle $(\rho=1$ and $0 \leq \theta<2 \pi$ ). (b) Solid curve: ${ }^{10} \log \left|\Re\left\{f-f_{p}^{40}\right\}\right|$ on the unit circle rim; dashed curve: ${ }^{10} \log \left|\Re\left\{f_{p}^{40}-f_{p Z}^{40}\right\}\right|$; dot-dashed curve: ${ }^{10} \log \left|\Re\left\{f-f_{Z}^{40}\right\}\right|$.

of $N$ from 0 up to $N_{\max }=30,50$, and 70 in the respective cases of Figs. 3(a)-3(c). Next, for each of the cases (a), (b), (c) in Fig. 3 , the $N$ used in Eq. (36) defining $f_{p Z}^{N, N_{1}}$ is fixed at $N_{\text {max }}$, and ${ }^{10} \log \delta_{3}$ is plotted (dashed curve) as a function of $N_{1}=0,1, \ldots, N_{\max }$. These are the following observations:

- the value assumed by ${ }^{10} \log \delta_{1}$ at $N=N_{\text {max }}$ is already assumed by ${ }^{10} \log \delta_{2}$ at $N=0,27,38$ in the respective cases of Figs. 3(a)-3(c),

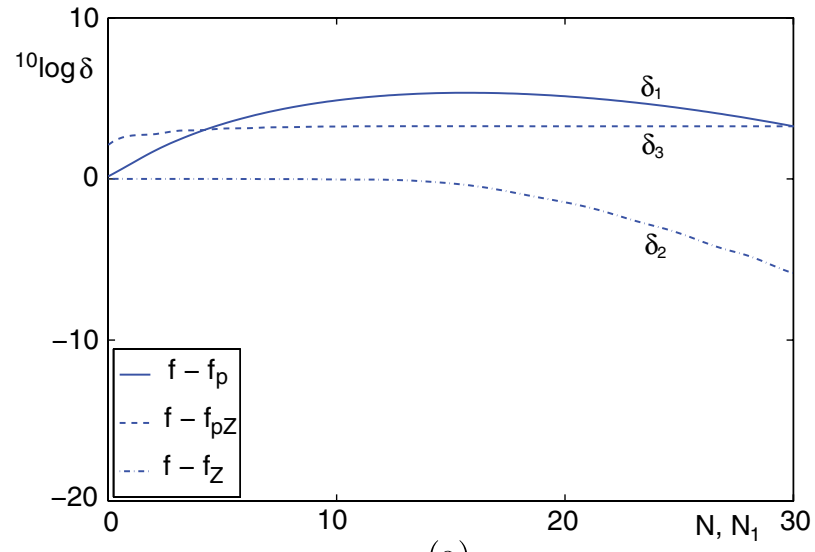

(a)
- the graphs of ${ }^{10} \log \delta_{1}$ and ${ }^{10} \log \delta_{2}$ saturate at a level -10 and -15 from $N=62$ and 45 onward, respectively,

- the values of ${ }^{10} \log \delta_{1}$ and ${ }^{10} \log \delta_{3}$ coincide at $N=N_{1}=N_{\max }$,

- ${ }^{10} \log \delta_{3}$ decreases slightly when $N_{1}$ is decreased below $N_{\text {max }}$ until the point $N_{1}=0,27,38$ is reached in the respective cases, where the graphs of ${ }^{10} \log \delta_{2}$ and ${ }^{10} \log \delta_{3}$ intersect, and these graphs practically coincide when $N_{1}$ is decreased further.

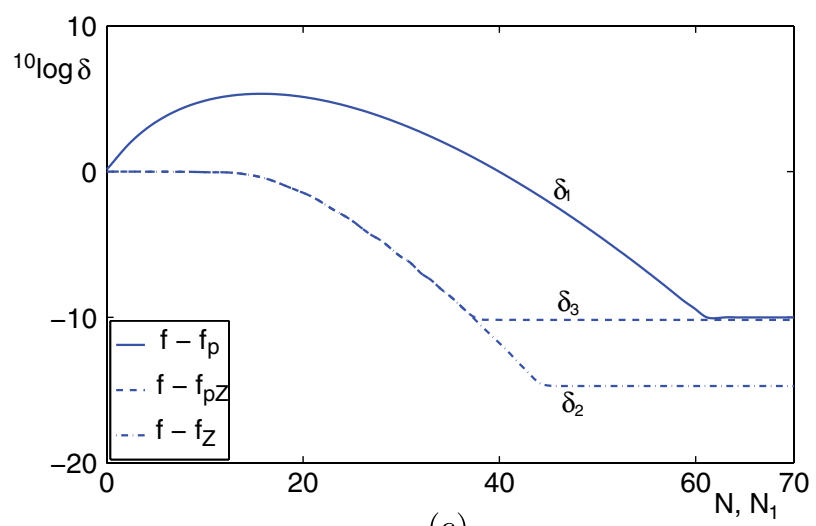

(c)

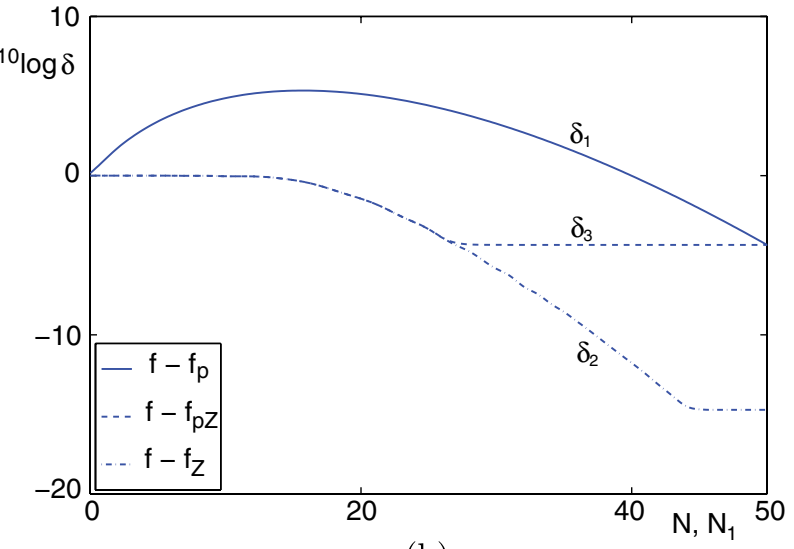

(b)

Fig. 3. Residual rms errors $\delta_{1}$ and $\delta_{2}$ for the representation of the exponential test function $[u=2.5, v=1.2$ in Eq. (27)] according to Eqs. (33) and (34), respectively, as a function of $N \leq N_{\max }=30,50$, and 70 in (a), (b), and (c), respectively. Furthermore, the residual rms error $\delta_{3}$ for the representation of the same test function according to Eq. (36) with $N=N_{\max }$ and $N_{1}=0,1, \ldots, N_{\max }$. 
The saturation matter for the graphs of ${ }^{10} \log \delta_{1}$ and ${ }^{10} \log \delta_{2}$ can be explained from the machine precision (equal to approximately 15 significant decimal positions when standard "double precision" arithmetic is used), from the fact that about 5 decimal places are lost when computing $f_{p}^{N}$ due to large values of $\left|a_{n m}\right|$, and the fact that $\left|c_{n m}\right|$ have reached a level of $10^{-15}$ from $n=45$ onward.

That the values of ${ }^{10} \log \delta_{1}$ and ${ }^{10} \log \delta_{3}$ coincide at $N=$ $N_{1}=N_{\text {max }}$ should be expected since $f_{p Z}^{N}$ and $f_{p Z}^{N, N}$ coincide with one another within machine precision.

An explanation of the last of the observed phenomena above is somewhat more subtle. Consider, instead of the quantity $\delta$ in Eq. (37), the analytically more tractable quantity

$$
I\left|f-f_{\text {app }}\right|^{2}=\frac{1}{\pi} \iint_{X^{2}+Y^{2} \leq 1}\left|f(X, Y)-f_{\text {app }}(X, Y)\right|^{2} \mathrm{~d} X \mathrm{~d} Y .
$$

We have from Eqs. (29) and (36) that

$$
f-f_{p Z}^{N, N_{1}}=\sum_{n, m, n>N_{1}} c_{n m} Z_{n}^{m}+\sum_{n_{1}, m_{1}, n_{1} \leq N_{1}}\left(c_{n_{1} m_{1}}-c_{n_{1} 0 m_{1} 0}^{N}\right) Z_{n_{1}}^{m_{1}} .
$$

Hence, by the orthogonality of the circle polynomials and the normalization in Eq. (ㅁ), we have

$$
\begin{aligned}
& I\left|f-f_{p Z}^{N, N_{1}-1}\right|^{2}-I\left|f-f_{p Z}^{N, N_{1}}\right|^{2} \\
& \quad=\frac{1}{N_{1}+1}\left(\sum_{n, m, n=N_{1}}\left|c_{n m}\right|^{2}-\sum_{n_{1}, m_{1}, n_{1}=N_{1}}\left|c_{n_{1} m_{1}}-c_{n_{1} 0 m_{1} 0}^{N}\right|^{2}\right)
\end{aligned}
$$

when $N_{1}$ decreases from $N_{\max }$, the second term in Eq. (40) dominates the first one until the $c_{N_{1} m}$ and $c_{N_{1} 0 m_{1} 0}^{N}$ involved have the same order of magnitude. Hence, the quantity in Eq. (40) is negative until then. When $N_{1}$ is decreased further, the error made in computing the $c_{N_{1} 0 m_{1} 0}^{N}$ as estimates of $c_{N_{1} m}$ is smaller than the magnitudes of the $c_{N_{1} m}$ themselves, and so the graphs of ${ }^{10} \log \delta_{2}$ and ${ }^{10} \log \delta_{3}$ coincide to an increasing extent with lower $N_{1}$.

\section{CONCLUSION}

Explicit expressions have been given for the coefficients of a double Zernike expansion of a function of three or four variables from its power series expansion. The conversion scheme for the expansion coefficients is exact and can be applied to the (real) aberration function of an optical imaging system. The conversion process is based on the calculation of the inner product of a power series term with a specific product of two Zernike polynomials, one defined on the pupil plane coordinates, and the other one on the image plane coordinates. Complex exponentials are used for the description of the azimuthal dependence of the Zernike polynomials. The advantages are an easier administration and the fact that the complex coefficients of a complex function can be calculated within the same framework as that for real functions.

The inner products are given in closed form and can be calculated up to machine precision for arbitrary high orders. Separate expressions have been derived for the frequently encountered optical system with rotational symmetry and for the more general system without this symmetry property. The order of Zernike polynomials is generally limited to those in the classical list of "Fringe-Zernike polynomials." In modern highquality imaging systems like lithographic projection lenses and very large astronomical telescopes with segmented subapertures, much higher orders are needed, either in measurement or in modeling. With the analysis presented in this paper, an optical aberration function in power series notation of high order can be conveniently and very accurately converted into the corresponding double Zernike expansion, avoiding the cumbersome administration and lengthy results from earlier work.

Numerical computations have shown that the Zernike expansion reproduces the function value given by the initial power series up to the machine precision. In the case of analytically given pupil functions, it is observed that the maximum degree of the obtained Zernike expansion can be substantially decreased, maintaining the same level of approximation of the initial function. The numerical exercises show that in this case a reduction in maximum degree of the Zernike expansion by a factor of typically two is feasible.

The conversion scheme can be applied not only to the optical aberration function itself but also to the complex exit pupil function $f=A \exp \{i \Phi\}$ with the amplitude function $A$ and the phase function $\Phi$ on the exit pupil given as a function of the position of the image point. Starting from the power series expansions of $A$ and $\Phi$, the power series expansion of $f$ is obtained by analytic means or with the aid of formal algebra. This power series expansion is then converted to a (double) Zernike expansion. With the aid of the complex coefficients of this expansion the complex amplitude distribution of the diffraction image is calculated in a semi-analytic way using the extended Nijboer-Zernike diffraction theory [34,35]; the analogous computation of high-numerical-aperture diffraction images is found in [32].

\section{APPENDIX A: INTEGRAL OF THE PRODUCT OF A MONOMIAL AND A RADIAL ZERNIKE POLYNOMIAL}

Here we follow the approach in [29] and [33] where, with the aid of Rodrigues' equation for the Jacobi polynomials, it is shown that

$$
R_{n}^{m}(\rho)=\frac{\rho^{-m}}{\left(\frac{n-m}{2}\right) !}\left\{\frac{d}{d\left(\rho^{2}\right)}\right\}^{\frac{n-m}{2}}\left\{\left(\rho^{2}\right)^{\frac{n+m}{2}}\left(\rho^{2}-1\right)^{\frac{n-m}{2}}\right\}
$$

with $m$ nonnegative and $n-m$ even and nonnegative. This expression is used for the calculation of the integral of Eqs. (14) and $(\underline{21})$,

$$
I=\int_{0}^{1} \rho^{a} R_{n}^{m}(\rho) \rho \mathrm{d} \rho .
$$

We put $(n-m) / 2=p,(n+m) / 2=q$, insert the Rodrigues expression in Eq. (A2), and with $\rho^{2}=x$ we obtain

$$
I=\frac{1}{2(p !)} \int_{0}^{1} x^{(a-m) / 2}\left\{\frac{d}{d x}\right\}^{p}\left[x^{q}(x-1)^{p}\right] \mathrm{d} x .
$$

By a single integration step by parts, we obtain

$$
I=\frac{1}{2(p !)}\left(\frac{a-m}{2}\right) \int_{0}^{1} x^{(a-m) / 2-1}\left\{\frac{d}{d x}\right\}^{p-1}\left[x^{q}(x-1)^{p}\right] \mathrm{d} x .
$$


After $p$ integrations by parts we have, using $q-p=m$,

$$
I=\frac{(-1)^{p}}{2(p !)}\left(\frac{a-m}{2}\right) \cdots\left(\frac{a-m}{2}-p+1\right) \int_{0}^{1} x^{(a+m) / 2}(x-1)^{p} \mathrm{~d} x .
$$

The remaining integral over $x$ is equally subjected to $p$ integrations by parts,

$$
I=\frac{(-1)^{2 p}}{2} \frac{\left(\frac{a-m}{2}\right) \cdots\left(\frac{a-m}{2}-p+1\right)}{\left(\frac{a+m}{2}+1\right) \cdots\left(\frac{a+m}{2}+p\right)} \int_{0}^{1} x^{(a+m) / 2+p} \mathrm{~d} x .
$$

We then obtain the final result

$$
I=\frac{\frac{1}{2}\left(\frac{a-m}{2}\right) \cdots\left(\frac{a-m}{2}-p+1\right)}{\left(\frac{a+m}{2}+1\right) \cdots\left(\frac{a+m}{2}+p+1\right)} .
$$

The derivation is valid as long as $(a-m) / 2-p>0$. However, both $I$ in Eq. (A2) and the right-hand side of Eq. (A7) depend analytically in $a$ with $\Re(a)>-m-2$. Therefore, the result of Eq. (A7) extends to this range by analyticity. Finally, the result of Eq. (A7) can be written in terms of $\Gamma$ functions as

$$
I=\frac{\frac{1}{2} \Gamma\left(\frac{a-m}{2}+1\right) \Gamma\left(\frac{a+m}{2}+1\right)}{\Gamma\left(\frac{a-m}{2}-p+1\right) \Gamma\left(\frac{a+m}{2}+p+2\right)},
$$

where the right-hand side vanishes when $a=m+2 p-2$, $m+2 p-4, \ldots, m$.

A numerically stable evaluation of $I$ in (A7) is based on the product representation

$$
I=\frac{1}{a+m+2 p+2} \prod_{j=0}^{p-1} \frac{a-m-2 j}{a+m+2 j+2} .
$$

The general factor of the product expression in (A9) is well behaved and $\leq 1$, and a numerically accurate calculation of $I$ is possible in standard double precision arithmetic for arbitrary high orders $n$ and $a$. For $p=0$, the multiple product is put equal to unity.

\section{APPENDIX B: DISCRETE FOURIER TRANSFORM COMPUTATION OF THE FOURIER COEFFICIENTS OF $\cos ^{n} \theta \sin ^{m} \theta$}

Assume that $f(\theta)$ is a $2 \pi$ periodic, integrable function of $\theta$, with Fourier series

$$
f(\theta)=\sum_{m_{2}=-\infty}^{+\infty} a_{m_{2}} \exp \left(i m_{2} \theta\right)
$$

The Fourier coefficients of $f$ can be approximated or computed by discretization of the Fourier integral

$$
a_{m_{1}}=\frac{1}{2 \pi} \int_{0}^{2 \pi} f(\theta) \exp \left(-i m_{1} \theta\right) \mathrm{d} \theta, \quad \text { integer } m_{1}
$$

Thus for any $S=1,2, \ldots$, we have from Eq. $\underline{(\mathrm{B} 1)}$

$$
\begin{aligned}
\frac{1}{S} \sum_{s=0}^{S-1} f\left(\frac{2 \pi s}{S}\right) \exp \left(-2 \pi i m_{1} \frac{s}{S}\right) \\
\quad=\frac{1}{S} \sum_{s=0}^{S-1} \sum_{m_{2}=-\infty}^{+\infty} a_{m_{2}} \exp \left[-2 \pi i\left(m_{2}-m_{1}\right) \frac{s}{S}\right] \\
\quad=\sum_{r=-\infty}^{+\infty} a_{m_{1}+r S},
\end{aligned}
$$

where it has been used that for integer $t$

$$
\sum_{s=0}^{S-1} \exp \left(2 \pi i t \frac{s}{S}\right)= \begin{cases}S, & t \text { multiple of } S \\ 0, & \text { otherwise }\end{cases}
$$

In the case that

$$
f(\theta)=\cos ^{n} \theta \sin ^{m} \theta,
$$

we have that $a_{m_{2}}=0$ in Eq. ( $\left.\underline{\mathrm{B} 1}\right)$ when $\left|m_{2}\right|>n+m$. Therefore, when $m_{1}$ is an integer with $\left|m_{1}\right| \leq n+m$ and $S>2(n+m)$, the series on the last line of Eq. (B3) has only one nonzero term, viz. the term with $r=0$. Hence we have then

$$
a_{m_{1}}=\frac{1}{S} \sum_{s=0}^{S-1} f\left(\frac{2 \pi s}{S}\right) \exp \left(-2 \pi i m_{1} \frac{s}{S}\right) .
$$

Now also note that $f(\theta)$ in Eq. ( $\underline{\mathrm{B} 5})$ is real, and so $a_{-m_{1}}=a_{m_{1}}$ for integer $m_{1}$. We thus conclude that all required numbers $I_{n m}^{m_{1}}$ of Eq. (19) can be obtained according to

$$
\begin{aligned}
I_{n m}^{m_{1}} & =\int_{0}^{2 \pi} \cos ^{n} \theta \sin ^{m} \theta \exp \left(-i m_{1} \theta\right) \mathrm{d} \theta=2 \pi a_{m_{1}} \\
& =\frac{2 \pi}{S} \sum_{s=0}^{S-1} \cos ^{n}\left(\frac{2 \pi s}{S}\right) \sin ^{m}\left(\frac{2 \pi s}{S}\right) \exp \left(-2 \pi i \frac{m_{1} s}{S}\right),
\end{aligned}
$$

for $m_{1}=0,1, \ldots, n+m, n+m+1,, \ldots, S-1$. Equation (B7) has the form of a discrete Fourier transform (DFT) on $S$ points applied to the function $f(\theta)$ in Eq. (B5) sampled at $\theta=2 \pi s / S$, $s=0,1, \ldots, S-1$. This DFT formula has a fast implementation, called FFT, in which all quantities $I_{n m}^{m_{1}}, 0 \leq m_{1} \leq$ $n+m$ for a given $n$ and $m$ are computed simultaneously, using only $O(S \ln S)$ operations and with very favorable roundoff error propagation.

The approach of evaluating azimuthal integrals using the DFT applies also for the double integral in Eq. (12); this integral can be written as

$$
2 \pi \delta_{m_{1}-m_{2}} \int_{0}^{2 \pi} \cos ^{m} \theta \exp \left(-i m_{1} \theta\right) \mathrm{d} \theta
$$

and the remaining integral is of the form of (B7).

\section{REFERENCES}

1. L. Seidel, "Über die Entwicklung der Glieder 3ter Ordnung welche den Weg eines ausserhalb der Ebene der Axe gelegene 
Lichtstrahles durch ein System brechender Medien bestimmen," Astr. Nach. 43, 289-304 (1856).

2. K. Schwarzschild, "Untersuchungen zur geometrischen Optik, I-II,” Abh. Königl. Ges. Wiss. Göttingen, Math. Phys. Kl, Neue Folge 4, 1-54 (1905).

3. F. Wachendorf, "Bestimmung der Bildfehler fünfter Ordnung in zentrierten optischen Systemen," Optik (Jena) 5, 80-122 (1949).

4. H. A. Buchdahl, Aberrations of Optical Systems (Dover Publications, 1968)

5. M. R. Rimmer, "Optical aberration coefficients," Ph.D. thesis (University of Rochester, 1963).

6. T. B. Andersen, "Automatic computation of optical aberration coefficients," Appl. Opt. 19, 3800-3816 (1980).

7. F. Bociort, T. B. Andersen, and L. H. J. F. Beckmann, "Highorder optical aberration coefficients: extension to finite objects and to telecentricity in object space," Appl. Opt. 47, 5691-5700 (2008).

8. J. L. Synge, Geometrical Optics, An Introduction to Hamilton's Method, Cambridge Tracts in Mathematics and Mathematical Physics (Cambridge University, 1937).

9. T. Smith, "The changes in aberrations when the object and stop are moved," Trans. Opt. Soc. 23, 139-153 (1922).

10. G. C. Steward, "Aberration diffraction effects," Phil. Trans. R. Soc. A 225, 131-198 (1926)

11. C. Carathéodory, "Geometrische Optik," in Ergebnisse der Mathematik und ihrer Grenzgebiete (Springer-Verlag, 1937), Vol. 4, no. 5.

12. C. H. F. Velzel and J. L. F. de Meijere, "Characteristic functions and the aberrations of symmetric optical systems. I. Transverse aberrations when the eikonal is given," J. Opt. Soc. Am. A 5, 246-250 (1988).

13. C. H. F. Velzel and J. L. F. de Meijere, "Characteristic functions and the aberrations of symmetric optical systems. II. Addition of aberrations," J. Opt. Soc. Am. A 5, 251-256 (1988).

14. C. H. F. Velzel and J. L. F. de Meijere, "Characteristic functions and the aberrations of symmetric optical systems. III. Calculation of eikonal coefficients," J. Opt. Soc. Am. A 5, 1237-1243 (1988).

15. H. H. Hopkins, Wave Theory of Aberrations (Clarendon Press, 1950).

16. A. E. Conrady, Applied Optics and Optical Design [Dover Publications, Part I (1957), Part II (1960)].

17. J. Sasián, "Theory of sixth-order wave aberrations," Appl. Opt. 49, D69-D95 (2010).

18. R. K. Tyson, "Conversion of Zernike aberration coefficients to Seidel and higher-order power-series aberration coefficients," Opt. Lett. 7, 262-264 (1982).
19. G. Conforti, "Zernike aberration coefficients from Seidel and higher-order power-series coefficients," Opt. Lett. 8, 407-408 (1983).

20. I. W. Kwee and J. J. M. Braat, "Double Zernike expansion of the optical aberration function," Pure Appl. Opt. 2, 21-32 (1993).

21. I. Agurok, "Double expansion of wavefront deformation in Zernike polynomials over the pupil and field-of-view of optical systems: lens design, testing, and alignment," Proc. SPIE 3430, 80-87 (1998).

22. T. Matsuyama and T. Ujike, "Orthogonal aberration functions for microlithographic optics," Opt. Rev. 11, 199-207 (2004).

23. K. P. Thompson, "Aberration fields in tilted and decentered optical systems," Ph.D. thesis (The University of Arizona, 1980).

24. I. Agurok, "Aberrations of perturbed and unobscured optical systems," Proc. SPIE 3779, 166-177 (1999).

25. K. P. Thompson, "Description of the third-order optical aberrations of near-circular pupil optical systems without symmetry," J. Opt. Soc. Am. A 22, 1389-1401 (2005).

26. K. P. Thompson, "Multinodal fifth-order optical aberrations of optical systems without rotational symmetry: spherical aberration," J. Opt. Soc. Am. A 26, 1090-1100 (2009).

27. T. Matsuzawa, "Image field distribution model of wavefront aberration and models of distortion and field curvature," J. Opt. Soc. Am. A 28, 96-110 (2011).

28. R. W. Gray, C. Dunn, K. P. Thompson, and J. P. Rolland, "An analytic expression for the field dependence of Zernike polynomials in rotationally symmetric optical systems," Opt. Express 20, 16436-16449 (2012).

29. B. R. A. Nijboer, "The diffraction theory of aberrations," Ph.D. thesis (Rijksuniversiteit Groningen, 1942), J. B. Wolters, Groningen, downloadable from www.nijboerzernike.nl.

30. A. J. E. M. Janssen and P. Dirksen, "Computing Zernike polynomials of arbitrary degree using the discrete Fourier transform," J. Eur. Opt. Soc. Rapid Pub. 2, 07012 (2007).

31. V. N. Mahajan, Optical Imaging and Aberrations: Part I, Ray Geometrical Optics, (SPIE Press, 1998), p. 156.

32. J. J. M. Braat, P. Dirksen, A. J. E. M. Janssen, and A. S. van de Nes, "Extended Nijboer-Zernike representation of the field in the focal region of an aberrated high-aperture optical system," J. Opt. Soc. Am. A 20, 2281-2292 (2003).

33. M. Born and E. Wolf, Principles of Optics, 7th expanded ed. (Cambridge University, 1999), pp. 909-910.

34. A. J. E. M. Janssen, "Extended Nijboer-Zernike approach for the computation of optical point-spread functions," J. Opt. Soc. Am. A 19, 849-857 (2002).

35. J. J. M. Braat, P. Dirksen, and A. J. E. M. Janssen, "Assessment of an extended Nijboer-Zernike approach for the computation of optical point-spread functions," J. Opt. Soc. Am. A 19, 858-870 (2002). 